1 Cambridge Institute of Therapeutic Immunology and Infectious Disease. Cambridge, UK

2 Cambridge University Hospitals, Cambridge, UK

3 Cambridge Institute for Medical Research, Cambridge, UK

Correspondence to: N J Matheson njm25@cam.ac.uk

Cite this as: BMJ 2021;375:n2388 http://dx.doi.org/10.1136/bmj.n2388

Published: 01 October 2021

\section{Mass testing of university students for covid-19}

\author{
The case for regular PCR testing of all students remains strong
}

Nicholas J Matheson, 1,2 Ben Warne, 1,2 Michael P Weekes, ${ }^{2,3}$ Patrick H Maxwell2, 3

People can clearly incubate and transmit SARS-CoV-2 in the absence of symptoms. ${ }^{1}$ Nonetheless, the value of mass testing (large scale asymptomatic screening to identify cases) has been contested. ${ }^{23}$ In principle, isolation of people with pre-symptomatic or asymptomatic SARS-CoV-2 infection will prevent further spread. In practice, it is less clear whether enough infectious people can be identified through screening to have a quantitatively important effect on transmission, and whether the direct benefits of enhanced case ascertainment are outweighed by direct or indirect costs. The debate is complicated by an absence of data from randomised controlled trials, and controversy about the suitability of lateral flow tests for this purpose. 45

Students in higher education are at increased risk of SARS-CoV-2 infection because of their shared accommodation, abundant social contacts, low priority for vaccination, and potential for vaccine hesitancy. ${ }^{67}$ At the same time, universities have been at the forefront of research on covid-19. It is therefore instructive to consider how these institutions have sought to control transmission among their students.

As well as promoting vaccination, testing students with symptoms, and contact tracing, many universities in the UK and North America have implemented programmes to screen for asymptomatic and pre-symptomatic cases using weekly or twice weekly laboratory based PCR tests. Data from these programmes are now available from institutional websites, preprints, and peer reviewed publications. What can they teach us about mass testing for SARS-CoV-2?

The evidence suggests it is possible to sustain high levels of adherence to regular, voluntary, asymptomatic screening using nose and throat swabs. ${ }^{8} 9$ University led testing programmes have been strongly supported by students, ${ }^{810} 11$ providing reassurance at a time when student mental health and wellbeing have been severely affected by the pandemic. ${ }^{812}$

The data also show that mass testing can markedly increase case ascertainment, including a substantial proportion of people who have yet to develop symptoms (pre-symptomatic infection). ${ }^{913-15}$ During certain stages of the pandemic, some universities detected more students with SARS-CoV-2 infection through asymptomatic screening than through symptomatic testing. ${ }^{14-16}$ Provided these students are supported to self-isolate, it is reasonable to infer a substantial reduction in ongoing transmission.

PCR testing seems ideally suited to regular screening of defined populations, since high test sensitivity minimises the risk of false negative results and samples are available for genomic sequencing. ${ }^{17}$ In a university context, laboratory and logistical infrastructure can be planned in advance, turnaround time minimised, and swab or sample pooling used to reduce costs and demands on testing capacity, particularly when incidence is low. ${ }^{915}{ }^{18}$ False positive results can often be reduced by a two-step testing strategy, whereby a positive screening test result is followed routinely by a confirmatory PCR test. ${ }^{9}$ Regular, frequent screening is essential to ensure that infected people are detected early, while they are still infectious, so that self-isolation is justified and effective.

What, then, are the remaining unknowns-and how can success be measured? Evidence about secondary behavioural changes, which may partially offset the benefits of enhanced case detection, remains limited. This is a particular concern for programmes based on lateral flow tests because false negative results are more common and good evidence of sustained adherence to twice weekly home testing is lacking. ${ }^{19}$ In addition, it is unclear how vaccination will affect participation in voluntary mass testing. Screening programmes must therefore monitor both participation rates (how many people are screened and how often) and the fraction of all cases ascertained by mass testing.

Countries with high levels of vaccination are generally rolling back non-pharmaceutical interventions designed to limit case numbers such as social distancing and face masks. At the same time, the relative benefits of identifying and isolating contacts have been reduced, because transmission rates are lower when index cases or their contacts have been vaccinated. ${ }^{20} 21$ Nonetheless, the emergence of new SARS-CoV-2 variants means that large outbreaks may still occur in vaccinated populations. $^{22}$

Compared with other interventions, asymptomatic screening offers several advantages. Critically, it is focused on the identification and isolation of cases rather than contacts; it need not affect the freedom of individuals (provided testing is informed and voluntary); and the costs of each programme are direct and quantifiable, with few indirect economic consequences.

As long as pandemic control measures are required, a strong argument exists for mass testing of populations at high risk of infection, such as students in higher education. Faced with spread of the delta variant, many universities have committed to continuing their programmes of regular PCR based screening of asymptomatic students. When prevalence declines, surveillance testing (regular screening of a fraction of the relevant population) 
and genomic sequencing to identify new variants of concern may be a proportionate response, and universities will again be ideal laboratories to test the coherence and effectiveness of these approaches.

Competing interests: We have read and understood BMJ policy on declaration of interests and have no interests to declare.

Provenance and peer review: Not commissioned; externally peer reviewed.

1 Buitrago-Garcia D, Egli-Gany D, Counotte MJ, etal. Occurrence and transmission potential of asymptomatic and presymptomatic SARS-CoV-2 infections: A living systematic review and meta-analysis. PLoS Med 2020;17:e1003346.

doi: 10.1371/journal.pmed.1003346. pmid: 32960881

2 Deeks JJ, Brookes AJ, Pollock AM. Operation Moonshot proposals are scientifically unsound. BMJ 2020;370:m3699. doi: 10.1136/bmj.m3699. pmid: 32963111

3 Mina MJ, Peto TE, García-Fiñana M, Semple MG, Buchan IE. Clarifying the evidence on SARS-CoV-2 antigen rapid tests in public health responses to COVID-19. Lancet 2021;397:1425-7. doi: 10.1016/S0140-6736(21)00425-6. pmid: 33609444

4 Deeks JJ, Raffle AE. Lateral flow tests cannot rule out SARS-CoV-2 infection. BMJ2020;371:m4787. doi: 10.1136/bmj.m4787. pmid: 33310701

5 Taylor-Phillips S, Dinnes J. Asymptomatic rapid testing for SARS-CoV-2. BMJ2021;374:n1733. doi: 10.1136/bmj.n1733. pmid: 34233894

6 Hill EM, Atkins BD, Keeling MJ, etal. Modelling SARS-CoV-2 transmission in a UK university setting.medRxiv 2021:2020.10.15.20208454. [Preprint.] doi: 10.1101/2020.10.15.20208454

7 Troiano G, Nardi A. Vaccine hesitancy in the era of COVID-19. Public Health 2021;194:245-51. doi: 10.1016/j.puhe.2021.02.025. pmid: 33965796

8 Blake H, Corner J, Cirelli C, etal. Perceptions and experiences of the University of Nottingham pilot SARS-CoV-2 asymptomatic testing service: a mixed-methods study. Int J Environ Res Public Health 2020;18:E188. doi: 10.3390/ijerph18010188. pmid: 33383781

9 Warne B, Metaxaki M, Fuller S, etal. Feasibility and efficacy of mass testing for SARS-CoV-2 in a UK university using swab pooling and PCR.Research Square 2021. [Preprint.] doi: 10.21203/rs.3.rs-520626/v1

10 Cox C, Ansari A, McLaughlin M, etal. Developing an ethical framework for asymptomatic COVID-19 testing programmes in higher education institutions. Wellcome Open Res 2021doi: 10.12688/wellcomeopenres.16807.1

11 Gillam TB, Cole J, Gharbi K, etal. Norwich covid-19 testing initiative pilot: evaluating the feasibility of asymptomatic testing on a university campus. J Public Health (Oxf) 2021;43:82-8. doi: 10.1093/pubmed/fdaa194. pmid: 33124664

12 National Union of Students. Coronavirus student survey phase III November 2020-mental health and wellbeing. https://www.nusconnect.org.uk/resources/coronavirus-and-students-phase3-study-mental-health-with-demographics-nov-2020.

13 Hamer DH, White LF, Jenkins HE, etal. Assessment of a covid-19 control plan on an urban university campus during a second wave of the pandemic. JAMA Netw Open 2021;4:e2116425. doi: 10.1001/jamanetworkopen.2021.16425. pmid: 34170303

14 Rennert L, McMahan C, Kalbaugh CA, etal. Surveillance-based informative testing for detection and containment of SARS-CoV-2 outbreaks on a public university campus: an observational and modelling study. Lancet Child Adolesc Health 2021;5:428-36. doi: 10.1016/S2352-4642(21)00060-2. pmid: 33751952

15 Denny TN, Andrews L, Bonsignori M, etal. Implementation of a pooled surveillance testing program for asymptomatic SARS-CoV-2 infections on a college campus - Duke University, Durham, North Carolina, August 2-October 11, 2020. MMWR Morb Mortal Wkly Rep 2020;69:1743-7. doi: 10.15585/mmwr.mm6946e1. pmid: 33211678

16 University of Cambridge. Data from the COVID-19 testing service. https://www.cam.ac.uk/coronavirus/stay-safe-cambridge-uni/data-from-covid-19-testing-service.

17 Aggarwal D, Warne B, Jahun AS, etal. Genomic epidemiology of SARS-CoV-2 in a UK university identifies dynamics of transmission. Research Square 2021. [Preprint.] doi: 10.21203/rs.3.rs-520627/v1

18 University of Edinburgh. TestEd. https://www.ed.ac.uk/tested-covid.

19 lacobucci G. Covid-19: Mass testing at UK universities is haphazard and unscientific, finds BMJ investigation. BMJ 2021;372:n848. doi: 10.1136/bmj.n848. pmid: 33789865

20 Harder T, Koch J, Vygen-Bonnet S, etal. Efficacy and effectiveness of COVID-19 vaccines against SARS-CoV-2 infection: interim results of a living systematic review, 1 January to 14 May 2021. Euro Surveill 2021;26. doi: 10.2807/1560-7917.ES.2021.26.28.2100563. pmid: 34269175

21 Harris RJ, Hall JA, Zaidi A, Andrews NJ, Dunbar JK, Dabrera G. Effect of vaccination on household transmission of SARS-CoV-2 in England. N Engl J Med 2021;385:759-60. doi: 10.1056/NEJMc2107717. pmid: 34161702

22 Brown CM, Vostok J, Johnson H, etal. Outbreak of SARS-CoV-2 infections, including covid-19 vaccine breakthrough infections, associated with large public gatherings - Barnstable County, Massachusetts, July 2021. MMWR Morb Mortal Wkly Rep 2021;70:1059-62.

doi: 10.15585/mmwr.mm7031e2. pmid: 34351882
This article is made freely available for use in accordance with BMJ's website terms and conditions for the duration of the covid-19 pandemic or until otherwise determined by BMJ. You may use, download and print the article for any lawful, non-commercial purpose (including text and data mining) provided that all copyright notices and trade marks are retained. 